\title{
H. Kleinert
}

\section{Quantum Field Theory of Black-Swan Events}

Received: date / Accepted: date

\begin{abstract}
Free and weakly interacting particles are described by a secondquantized nonlinear Schrödinger equation, or relativistic versions of it. They describe Gaussian random walks with collisions. By contrast, the fields of strongly interacting particles are governed by effective actions, whose extremum yields fractional field equations. Their particle orbits perform universal Lévy walks with heavy tails, in which rare events are much more frequent than in Gaussian random walks. Such rare events are observed in exceptionally strong windgusts, monster or rogue waves, earthquakes, and financial crashes. While earthquakes may destroy entire cities, the latter have the potential of devastating entire economies.
\end{abstract}

\section{Introduction}

Since the bestselling book "The Black Swan" by N.N. Taleb 11, the "disproportionate role of high-profile, hard-to-predict, and rare events that are beyond the realm of normal expectations in history, science, finance, and technology", has moved into public awareness, thereby contrasting previous bestesellers focusing on Gaussian distributions 3. Since the last financial crash and the recent "flash crash" 4] that has appeared in 2010 [4] with the approach of nanosecond-trading in stockmarkets, the dangers of such events for the world economy have become so worrisome that also politicians begin to get worried.

H. Kleinert

Institut für Theoretische Physik, Freie Universität Berlin, 14195 Berlin, Germany Tel.: +49-15-151958789

E-mail: h@klnrt.de 14195 Berlin 


\section{Quantum Field Theory}

The purpose of this lecture is to incorporate them into our present description of particle physics. The quantum-mechanical phenomena of fundamental particles is explained with high accuracy by Schrödinger theory. The wave equation for many particles can convenienty be reformulated as a secondquantized field theory, with an action that is the sum of quadratic and an interacting term

$$
\mathcal{A}=\mathcal{A}_{2}+\mathcal{A}_{\text {int }}
$$

where the term $\mathcal{A}_{2}$ has typically the form

$$
\mathcal{A}_{2}=\int d^{D} x d t \psi^{*}(\mathbf{x}, t)\left[i \partial_{t}+\hbar^{2} \nabla^{2} / 2 m-V(\mathbf{x})\right] \psi(\mathbf{x}, t),
$$

with $D$ being the space timension, $m$ the mass, and $V(\mathbf{x})$ some external potential. The interaction term $\mathcal{A}_{\text {int }}$ may be approximated in molecular systems by a fourth-order term in the field

$$
\mathcal{A}_{\text {int }}=\frac{1}{2} \int d^{D} x d^{D} x^{\prime} d t \psi^{*}\left(\mathbf{x}^{\prime}, t\right) \psi^{*}(\mathbf{x}, t) V_{12}\left(\mathbf{x}, \mathbf{x}^{\prime}\right) \psi(\mathbf{x}, t) \psi\left(\mathbf{x}^{\prime}, t\right),
$$

where $V_{12}\left(\mathbf{x}, \mathbf{x}^{\prime}\right)$ is some two-body potential.

If relativistic velocities are present, the field is generalized to a scalar Klein-Gordon field, or a quantized Dirac field. In molecular physics, the fourth-order term is due to the exchange of a minimally coupled quantized photon field and is proportional to $e^{2}$, where $e$ is the electric charge. The field equations may be studied with any standard method of quantum field theory, and corrections can be derived using perturbation theory in powers of $\alpha \equiv e^{2} / \hbar \approx 1 / 137$. Since $\alpha$ is very small, this appeoch is quite successful.

If time is continued analytically to imaginary values $t=i \tau$, one is faced with the so-called Euclidean version of quantum field theory. Then perturbation theory may be understood as developing a theory of particle physics from an expansion around Gaussian random walks. Indeed, the relativistic scalar free-particle propagator of mass $m$ in $D+1$-dimensional euclidean energy-momentum space $p^{\mu}=\left(\mathbf{p}, p_{4}\right)$, has the form

$$
G(p)=\frac{1}{\mathbf{p}^{2}+p_{4}^{2}+m^{2}}=\int_{0}^{\infty} d s e^{-s m^{2}} e^{-s\left(\mathbf{p}^{2}+p_{4}^{2}\right)},
$$

where the energy has been continued analytically to $p_{4}=-i E$. The Fourier transform of $e^{-s\left(\mathbf{p}^{2}+p_{4}^{2}\right)}$ is the distribution of Gaussian random walks of length $s$ in $D+1$ euclidean dimensions

$$
P\left(\mathbf{x}, x_{4}\right)=(4 \pi s)^{-(D+1) / 2} e^{-\left(|\mathbf{x}|^{2}+x_{4}^{2}\right) / 4 s},
$$

which makes the propagator (4) a superposition of such walks with lenghts distributed like $e^{-s m^{2}}$ [5, 6, 7, This propagator is the relativistic version of the free-field propagator of the action (2). The second-quantized field theory 
described by (11) accounts for grand-canical ensembles of orbits with their two-body interactions 8].

Gaussian random walks are a natural and rather universal starting point for many stochastic processes. For instance, they form the basis of the most important tool in the theory of financial markets, the Black-Scholes option price theory [9] (Nobel Prize 1997), by which a portfolio of assets is hoped to remain steadily growing through hedging. In fact, the famous central-limit theorem permits us to prove that many independent random movements of finite variance always pile up to display a Gaussian distribution [10.

However, since the last stock market crash and the still ongoing financial crisis it has become clear that realistic distributions belong to a more general universality class, the so-called Lévy stable distribution. They are the univarsal results of a pile up of random movements of infinite variance [11. They account for the fact that rare events, which initiate crashes, are much more frequent than in Gaussian distributions. These are events in the socalled Lévy tails $\propto 1 /|x|^{1+\lambda}$ of the distributions, whose description requires a Hamiltonian [7]

$$
H=\operatorname{const}\left(p^{2}\right)^{\lambda / 2}
$$

Such tail-events are present in the self-similar distribution of matter in the universe 12, 13, 14, in velocity distributions of many body sytems with longrange forces [15], and in the distributions of windgusts [16] and earthquakes [17, with often catastrophic consequences. They are a consequence of rather general maximal entropy assumptions [18. In the limit $\lambda \rightarrow 2$, the Lévy distributions reduce to Gaussian distributions.

\section{Strong-Coupling Quantum Field Theory}

At this point we observe that such distributions occur quite naturally also in many-particle systems, provided the interactions are very strong [19]. They have been observed in numerous experiments at second-order phase transitions. The most accurate measurement of this type was done in a satellite (the so called Infrared Atronomical Satellite IRAS) by studying the singularity of the specific heat of superfluid ${ }^{4} \mathrm{He}$ near the critical temperature [20]. The observation agreed extremely well with the theoretical strong-coupling prediction 21.

The field of a strongly interacting $N$-body system is usually a multivalued function. Singularities perforate the space via vortex lines (for instance in type II superconductors or in superfluid ${ }^{4} \mathrm{He}$ ), or via line-like defects in the displacement field of a world-crystal formulation of Einstein(-Cartan) gravity [22]. If the positions of two particles are exchanged, one obtains a factor +1 for bosons or -1 for electrons. In two dimensions, one may even obtain a general phase $e^{i \phi}$ (anyons) 25].

A strongly interacting field system has a conformally invariant Green function $23,24,25$.

$$
G\left(\mathbf{p}, p_{4}\right)=\left[p_{4}^{1-\gamma} \phi\left(\mathbf{p}^{2} / p_{4}^{z}\right)\right]^{-1}
$$


If the dimension $D$ differs only by a very small amount $\epsilon$ from the critical dimension $D_{c}$, where the theory is scale-invariant, i.e., $D=D_{c}+\epsilon$, then $\gamma$ is of order $\epsilon$ and $z$ differs from unity by a similar amount. Such a power behavior is assured near $D_{c}$ if the Gell-Mann-Low function [26] has an infrared-stable fixed point in the renormalization flow of the coupling constant. Very close to the critical dimension, a lowest approximation to $G\left(\mathbf{p}, p_{4}\right)$ is

$$
G\left(\mathbf{p}, p_{4}\right)=\left\{p_{4}^{1-\gamma}\left[1+D_{\lambda}\left(\mathbf{p}^{2} / p_{4}^{z}\right)^{\lambda / 2}\right]\right\}^{-1},
$$

where $\lambda$ is close to 2 , and $D_{\lambda}$ is a generalization of the diffusion constant in the Fokker-Planck equation.

Time-independent propagators involve the limit $p_{4} \rightarrow 0$, where the correlation function behaves like

$$
G(\mathbf{p}, 0) \propto|\mathbf{p}|^{-2+\eta} .
$$

The index $\eta$ is the anomalous dimension of the field, which is also of order $\epsilon$. The existence of this limit in (8) fixes the scaling relation

$$
\lambda=(2-\gamma) / z=2-\eta \text {. }
$$

See Appendix for the calculation of the exponents to order $\epsilon$. The Green function (8) determines the probability distribution of particle after a time $t$ via the doubly fractional Fokker-Planck equation

$$
\left[\hat{p}_{4}^{1-\gamma}+D_{\lambda}\left(\hat{\mathbf{p}}^{2}\right)^{\lambda / 2}\right] P(\mathbf{x}, t)=\delta(t) \delta^{(D)}(\mathbf{x}),
$$

where $\hat{p}_{4} \equiv \partial_{t}, \hat{\mathbf{p}} \equiv i \partial_{\mathbf{x}} \equiv i \boldsymbol{\nabla}$. A convenient definition of the fractional derivatives uses the same formula as in the dimensional continuation of Feynman diagrams $\left(-\nabla^{2}\right)^{\lambda / 2}=\Gamma[\lambda / 2]^{-1} \int d \sigma \sigma^{-\lambda / 2-1} e^{\sigma \nabla^{2} / 2}$ [27, 28]. The solution of (11) is given in the literature [29] and reads

$$
\frac{t^{-\gamma}}{\pi^{D / 2}|\mathbf{x}|^{D / 2}} H_{2,3}^{2,1}\left(\left.\frac{|\mathbf{x}|^{\lambda}}{2^{\lambda} D_{\lambda} t^{1-\gamma}}\right|_{(1,1),(D / 2, \lambda / 2) ;(1, \lambda / 2)} ^{(1,1) ;(1-\gamma, 1-\gamma)}\right),
$$

where $H_{2,3}^{2,1}$ is a Fox $H$-function [30]. In the limits $\gamma=0$ and $\lambda=2$, this reduces to the standard quantum mechanical Gaussian expression $\left(4 \pi D_{\lambda} t\right)^{-D / 2}$ $e^{-|\mathbf{x}|^{2} / 4 D_{\lambda} t}$. For $\gamma=0, \lambda=1$, the result is

$$
P(\mathbf{x}, t)=\frac{D_{\lambda} t}{\pi^{(D+1) / 2}|\mathbf{x}|^{D+1}} H_{1,1}^{1,1}\left(\left.\frac{D_{\lambda}^{2} t^{2}}{|\mathbf{x}|^{2}}\right|_{(0,1)} ^{(1 / 2-D / 2,1)}\right),
$$

which is simply the Cauchy-Lorentz distribution function

$$
\left[\Gamma(D / 2+1 / 2) / \pi^{(D+1) / 2}\right] D_{\lambda} t /\left[\left(D_{\lambda} t\right)^{2}+|\mathbf{x}|^{2}\right]^{D / 2+1 / 2} .
$$

The probability (11) may be calculated from the doubly fractional canonical path integral over fluctuating orbits $t(s), \mathbf{x}(s) p_{4}(s), \mathbf{p}(s)$ viewed as functions of some pseudotime $s$ [31:

$$
\left\{\mathbf{x}_{b} t_{b} s_{b} \mid \mathbf{x}_{a} t_{s} s_{a}\right\}=\int \mathcal{D} \mathbf{x} \mathcal{D} t \mathcal{D} \mathbf{p} \mathcal{D} p_{4} e^{-\mathcal{A}},
$$


where $\mathcal{A}$ is the euclidean action of the paths $t(s), \mathbf{x}(s)$ :

$$
\mathcal{A}=\int d s\left[i\left(\mathbf{p x}^{\prime}-i p_{4} t^{\prime}\right)-\mathcal{H}\left(\mathbf{p}, p_{4}\right)\right]
$$

Here $t^{\prime}(s) \equiv d t(s) / d s, \mathbf{x}^{\prime}(s) \equiv d \mathbf{x}(s) / d s$, and $\mathcal{H}\left(\mathbf{p}, p_{4}\right)=p_{4}^{1-\gamma}+D_{\lambda}\left(\hat{\mathbf{p}}^{2}\right)^{\lambda / 2}$. At each $s$, the integrals over the components of $\mathbf{p}(s)$ and $p_{4}(s)$ run from $-\infty$ to $\infty$, whereas those over $p_{4}(s)$ run from $-i \infty$ to $i \infty$. At the end we obtain $P(\mathbf{x}, t)$ from the integral $\int_{0}^{\infty} d s\{\mathbf{x} t s \mid \mathbf{0} 00\}$.

If $\gamma=0$, the path integral over $p_{4}(s)$ yields the functional $\delta\left[t^{\prime}(s)-1\right]$, which brings (14) to the canonical path integral

$$
\left(\mathbf{x}_{b} t_{b} \mid \mathbf{x}_{a} t_{a}\right)=\int \mathcal{D} \mathbf{x} \mathcal{D} \mathbf{p} e^{-\mathcal{A}^{\prime}}
$$

with

$$
\mathcal{A}^{\prime}=\int d t\left[i \mathbf{p} \dot{\mathbf{x}}-D_{\lambda}\left(\hat{\mathbf{p}}^{2}\right)^{\lambda / 2}\right]
$$

Now $P(\mathbf{x}, t)=(\mathbf{x} t \mid \mathbf{0} 0)$ satisfies the ordinary fractional Fokker-Planck equation

$$
\left[\hat{p}_{4}+D_{\lambda}\left(\hat{\mathbf{p}}^{2}\right)^{\lambda / 2}\right] P(t, \mathbf{x})=\delta(t) \delta^{(D)}(\mathbf{x})
$$

This has been discussed at length in recent literature 32 .

At this place it is worth mentioning that the probability can be written as a superposition $\int_{0}^{\infty}(d \sigma / \sigma) f_{\lambda}\left(\sigma t^{-2 / \lambda}\right) P_{\mathrm{G}}(\sigma, \mathbf{x})$ of Gaussian distributions $P_{\mathrm{G}}(\sigma, \mathbf{x})=(4 \pi \sigma)^{-D / 2} e^{-\mathbf{x}^{2} / 4 \sigma}$ with weight

$$
f_{\lambda}(\sigma)=S_{D} \sum_{n=1}^{\infty} \frac{(-1)^{n} \sigma^{-n \lambda / 2}}{(n+1) ! \Gamma(D-1-n \lambda / 2)} D_{\lambda}^{n / \lambda},
$$

where $S_{D}=2 \pi^{D / 2} / \Gamma(D / 2)$ is the surface of a sphere in $D$ dimensions.

\section{Stochastic Duru-Kleinert Transformation}

If $\gamma \neq 0$, the above functional $\delta$-function is softened, and the relation between the pseudotime $s$ and the physical time becomes stochastic. It is governed by the probability distribution that solves the path integral the

$$
\left.\left\{t_{b} s_{b} \mid t_{a} s_{a}\right\}=\int \mathcal{D} t \mathcal{D} p_{4} \exp \left\{\int d s\left[p_{4} t^{\prime}-p_{4}^{1-\gamma}\right)\right]\right\} .
$$

For imaginary $p_{4}=-i E$, we define a noise Hamiltonian $\tilde{H}(\eta)$ which has the property that 31,33

$$
e^{-p_{4}^{1-\gamma}}=\int_{-\infty}^{\infty} d \eta e^{-p_{4} \eta-\tilde{H}(\eta)}
$$


The inverse of the Fourier integral yields the noise probability $P(\eta)=$ $\int_{-i \infty}^{i \infty} d p_{4} e^{p_{4} \eta-p_{4}^{1-\gamma}}$, and a probability functional [34]:

$$
P[\eta] \equiv e^{-\int d s \tilde{H}(\eta)}=\int \mathcal{D} p_{4} \exp \left[\int d s\left(p_{4} \eta-p_{4}^{1-\gamma}\right)\right] .
$$

Using this we may solve the stochastic differential equation of the Langevin type

$$
t^{\prime}(s)=\eta(s),
$$

in which the noise $\eta(s)$ has a zero expectation value for each $s$, and the correlation functions for $n=2,4,6, \ldots$ :

$$
\left\langle\eta\left(s_{1}\right) \ldots \eta\left(s_{2 n}\right)\right\rangle \equiv \int \mathcal{D} \eta \eta\left(s_{1}\right) \ldots \eta\left(s_{2 n}\right) P[\eta] .
$$

If $\gamma=0$, the solution of (22) is $P[\eta]=\delta[\eta(s)-1]$, implying that $\eta(s)$ ceases to fluctuate, and (23) becomes $t^{\prime}(s) \equiv 1$, so that $t \equiv s$.

In the past, many nontrivial Schrödinger equations (for instance that of the $1 / r$-potential) have been solved with path integral methods by reformulating them on the pseudotime axis $s$, that is related to the time $t$ via a space-dependent differential equation $t^{\prime}(s)=f(x(t))$. This method, invented by Duru and Kleinert 35 to solve the path integral of the hydrogen atom, has recently been applied successfully to various Fokker-Planck equations [36, 37. The stochastic differential equation (23) may be seen as a stochastic version of the Duru-Kleinert transformation that promises to be a useful tool to study non-Markovian systems.

Certainly, the solutions of Eq. (18) can also be obtained from a stochastic differential equation

$$
\dot{\mathbf{x}}=\boldsymbol{\eta},
$$

whose noise is distributed with a fractional probability

$$
P[\boldsymbol{\eta}]=\int \mathcal{D}^{D} x e^{\int d t\left(i \mathbf{p} \cdot \boldsymbol{\eta}-D_{\lambda}\left(\mathbf{p}^{2}\right)^{\lambda / 2}\right)} .
$$

\section{Experimental Consequences}

Experimentally, a system with in the strong-coupling limit can be produced by forming a Bose-Einstein condensate (BEC) in a magnetic field whose strength is tuned to a Feshbach resonance 38 of the two-particle interaction. In a BEC, the four-field term in the interaction (3) is local and parametrized by $V_{12}(\mathbf{x}, \mathbf{x}) \propto g \delta\left(\mathbf{x}-\mathbf{x}^{\prime}\right)$. At the Feshbach resonance, the bare coupling strength $g$ goes to infinity 39 , and the renormalized coupling $g_{R}$, multiplied by $6 \mu^{-\epsilon} /(4 \pi)^{2}$, converges to a fixed point $g^{*} \approx 0.503$ [see Fig. 17.1 in Ref. [24]), where $\mu$ is some mass scale.

The theoretical tool to describe the physics in this regime is the effective action $\Gamma\left[\Psi, \Psi^{*}\right]$. This a functional of the classical expectation values of 
the quantum fields $\Psi(t, \mathbf{x}) \equiv\langle\psi(t, \mathbf{x})\rangle$, and contains all information of the full quantum theory [24,40. It is the Legendre transform of the generating functional $Z\left[\eta, \eta^{*}\right]=\int \mathcal{D} \psi \mathcal{D} \psi^{*} e^{-\mathcal{A}-\eta^{*} \psi-\eta \psi^{*}}$ of the full quantum theory, and is extremal on the physical field expections. All its vertex functions can be found from the functional derivatives of $\Gamma\left[\Psi, \Psi^{*}\right]$. In the strong-coupling limit, the effective interaction changes the interaction (3) to an anomalous power law $\Gamma^{\mathrm{int}}\left[\Psi, \Psi^{*}\right]=\left(g_{c} / 2\right) \int d t d^{D} x|\Psi(t, \mathbf{x})|^{\delta+1}$. The power $\delta$ is a critical exponent that is measured experimentally by the relation $B=|\Psi|^{\delta}$. Its value is determined by $\eta$ via the so-called hyperscaling relation [41] $\delta=(D+2-\eta) /(D-2+\eta)$. The value of $g_{c}$ is related to the critical value $g^{*} \approx 0.503$ by $g_{c} \mu^{-\eta D /(D-2+\eta)}=\left(2 g^{*}\right)^{(\delta-1) / 2}(4 \pi)^{2} / 24 \approx 6.7$. As a possible application we may study the behavior of a triangular lattice of vortices which form in a rotating Bose-Einstein condensate [42, and letting the magnetic field approach a Feshbach resonance.

The results may then be compared with a calculation based on a new field equation that generalizes the famous Gross-Pitaevskii equation [4]

$$
\left[\hat{E}-\frac{1}{2 m} \hat{\mathbf{p}}^{2}-g|\Psi(t, \mathbf{x})|^{2}\right] \Psi(t, \mathbf{x})=0 .
$$

The new equation is obtained by extremizing the effective action $\Gamma\left[\Psi, \Psi^{*}\right]=$ $\Gamma_{0}\left[\Psi, \Psi^{*}\right]+\Gamma^{\operatorname{int}}\left[\Psi, \Psi^{*}\right]$, where

$$
\Gamma_{0} \equiv \int d t d^{D} x \Psi^{\dagger}(t, \mathbf{x})\left[\hat{E}^{1-\gamma}-D_{\lambda}\left(\hat{\mathbf{p}}^{2}\right)^{\lambda / 2}\right] \Psi(t, \mathbf{x})
$$

By forming $\delta \mathcal{A}^{\text {eff }} / \delta \Psi^{\dagger}(t, \mathbf{x})$, we obtain what may be called the fractional Gross-Pitaevskii equation:

$$
\left[\hat{E}^{1-\gamma}-D_{\lambda}\left(\hat{\mathbf{p}}^{2}\right)^{1-\eta / 2}-\frac{\delta+1}{4 \mu^{\eta}} g_{c}|\Psi(t, \mathbf{x})|^{\delta-1}\right] \Psi(t, \mathbf{x})=0 .
$$

The fractional Schrödinger equation has many problems, such as the nonvalidity of the quantum superposition law, the violation of unitarity of the time evolution, and the violation of probability conservation which can produce nonsensical probabilities $>1$ 32. However, these problems exist only if we restrict ourselves to the free effective action (28), but this is meaningless, since the entire theory is only defined by the effective action in the strongcoupling limit - and this contains necessarily additional nonquadratic terms. Hence it does not possess free quasiparticles as in the time-honored Landau theory of Fermi liquids 44. There is always an interaction that invalidates the standard discussion of Schrödinger equations. In fact, the theory of high$T_{c}$ superconductivity must probably be built as a true strong-coupling theory of this type with electrons being non-Fermi liquids [44.

The relativistic version of the entire discussion is simpler since it is based on the euclidean Green function (9) in which $\mathbf{p}$ denotes the $D$-1-dimensional vectors $\left(\mathbf{p}, p_{4}\right)$. The Fourier transform is the distribution fulfilling the FokkerPlanck equation

$$
\left[\partial_{s}+\left(\hat{\mathbf{p}}^{2}\right)^{1-\eta / 2}\right] P(s, \hat{\mathbf{x}})=\delta(s) \delta^{(D+1)}(\mathbf{x}) .
$$


and possessing the path integral representation

$$
P(s, \hat{\mathbf{x}})=\int \mathcal{D} \mathbf{x} \mathcal{D} \mathbf{p} e^{\int d s\left[i \mathbf{p} \dot{\mathbf{x}}-\left(\hat{\mathbf{p}}^{2}\right)^{1-\eta / 2}\right]} .
$$

The $\epsilon$-expansion is now around $D_{c}=4$ in powers of $\epsilon=-\left(D-D_{c}\right)$. The critical exponent $\eta$ is small of order $\epsilon^{2}: \eta=\epsilon^{2} / 50+\cdots \approx 0.04$. It can be ignored for $\epsilon=1$. The power $\delta$ in the interaction is $3+\epsilon+23 \epsilon^{2} / 50+\cdots \approx 4.76$ [45].

The time-independent fractional Gross-Pitaevskii equation reads now

$$
\left[\left(\hat{\mathbf{p}}^{2}\right)^{1-\eta / 2}+\frac{\delta+1}{4 \mu^{\eta}} g_{c}|\Psi(\mathbf{x})|^{\delta-1}\right] \Psi(\mathbf{x})=0,
$$

with $g_{c} \approx 27$. For a $d=D-1$-dimensional vortex in $D=3$ dimensions, it is solved by $\tilde{\Psi}(\mathbf{x})=a\left|\mathbf{x}_{\perp}\right|^{-A}$ with $A=(2-\eta) /(\delta-1)=D / 2-1+\eta / 2 \approx 1 / 2$ and for $\mu=1$ : $\left[(\delta+1) a^{\delta-1} / 4\right] g_{c}=-{ }^{d} c_{\lambda+A-d} c_{A-d}^{-1} \approx 0.2, \lambda=2-\eta[28$.

Let us study the appearance of a reduced mass $\hat{m}^{2} \propto\left(1-r^{2}\right)$ in the trap. In the effective action, it will appear in (32) in the form $\mu^{2-\eta}\left(\hat{m}^{2}\right)^{\nu(2-\eta)}$ $\times f\left(|\Psi|^{2} /\left(\hat{m}^{2}\right)^{2 \beta}\right)$ multiplied by Taylor series of $f(x)$ with $\left.x \equiv|\Psi|^{2} /\left(\hat{m}^{2}\right)^{2 \beta}\right)$ (note that $\nu(2-\eta)=1+\frac{\epsilon}{5}+\cdots \approx 1.3$ ). For small $\hat{m}^{2}$, this may be resummed to a Widom type expression $\left[(\delta+1) / 4 \mu^{\eta}\right] g_{c}|\Psi|^{\delta-1} w\left(\hat{m}^{2} /|\Psi|^{1 / \beta}\right)$ [24. This explains the earlier-stated steeper falloff $|\Psi|^{2} \propto\left(\hat{m}^{2}\right)^{2 \beta}$ of the density profiles in Fig. 1. The Widom function $w\left(\hat{m}^{2} /|\Psi|^{1 / \beta}\right)$ can be expanded as 1 plus a power series in $\xi^{-\omega}|\Psi|^{-\omega \nu / \beta}$, which contains the Wegner critical exponent $\omega \approx 0.8$ that governs the approach to scaling [46]. Thereby the interaction term $|\Psi|^{\delta-1}$ is modified to $|\Psi|^{\delta-1}\left(1+\right.$ const $\left.\times \xi^{-\omega}|\Psi|^{-\omega \nu / \beta}\right)$. Similarly, the kinetic term $\left(\hat{\mathbf{p}}^{2}\right)^{1-\eta / 2}$ in (27), (32) will receive $\omega$-dependent corrections, and become $\left(\hat{\mathbf{p}}^{2}\right)^{1-\eta / 2}\left[1+\xi^{-\omega}\left(\hat{\mathbf{p}}^{2}\right)^{-\omega / 2}+\ldots\right]$.

To compare our theory with experimental data, we must study the BEC in the scale-invariant strong-coupling limit. This is reached either by going to the temperature $T_{c}$ of the second-order phase transition, or by raising the magnetic field $B$ towards the field strength $B_{c}$ of a Feshbach resonance [47. Then the coherence length $\xi$ grows like $\xi \propto|t|^{-\nu}$ where $\nu \approx 2 / 3$ [24, 48, and $t \equiv 1-T / T_{c}$ or $t \equiv 1-B / B_{c}$ (38]). If the BEC is enclosed in a weak harmonic trap, this adds in the brackets of (27) a term $\propto|\mathbf{x}|^{2}=R^{2}$. This is normally observed by the condensate density going to zero linearly like $1-r^{2} \equiv 1-R^{2} / R_{\mathrm{b}}^{2}$ near the border $R_{\mathrm{b}}$ (in the Thomas-Fermi approximation) [49. For $B$ near $B_{c}$ (or $T$ near $T_{c}$ ), however, the anomalous power $\delta$ will lead to the steeper approach to zero $\left(1-r^{2}\right)^{2 \beta}$ where $2 \beta \equiv \nu(D-2+\eta)=$ $1-3 \epsilon / 10+\cdots \approx 0.7$, plotted in Fig. 1 , as will be shown immediately. In addition, the central region is depleted.

\section{Summary}

Summarizing we have seen that a many-body system with strong couplings between the constituents satisfies a more general form of the Schrödinger equation, in which the momentum and the energy appear with a power different from $\lambda=2$ and $\gamma=0$, respectively. The associated Green function 

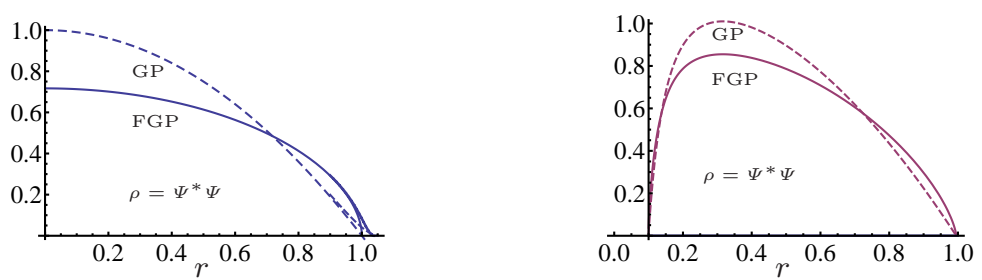

Fig. 1 Condensate density from Gross-Pitaevskii equation (27) (GP,dashed) and its fractional version 29 (FGP), both in Thomas-Fermi approximation where the gradients are ignored. The FGP-curve shows a marked depletion of the condensate. On the right hand, a vortex is included. The zeros at $r \approx 1$ will be smoothened by the gradient terms in (32), as indicated in the left-hand figure.

can be written as a path integral over fluctuating time and space orbits that are functions of some pseudotime $s$. This is a Markovian object, but non-Markovian in the physical time $t$ that is related to $s$ by a stochastic differential equation of the Langevin type. The particle distributions can also be obtained by solving a Langevin type of equation in which the noise has correlation functions whose probability distribution is specified.

Appendix: The lowest-order critical exponents can be extracted directly from the one-loop-corrected inverse Green function $G^{-1}(E, \mathbf{p})$ in $D=2+\epsilon$ dimensions after a minimal subtraction of the $1 / \epsilon$-pole at [50]:

$$
E-\mathbf{p}^{2}+a\left(\frac{1}{3} \mathbf{p}^{2}-E\right)^{D-1}
$$

For $\mathbf{p}=0$, this has a power $-(-E)^{1-a \epsilon}$, so that $\gamma=a \epsilon$. For $E=0$, on the other hand, we obtain $\left(-\mathbf{p}^{2}\right)^{1-a \epsilon / 3}$, so that $(1-\gamma) / z-1 \approx \gamma / 3$.

Note ADDED IN PROOF: While this lecture was being refereed, further progress has been made in the theory by H. Kleinert, Effective Action and Field Equation for BEC from Weak to Strong Couplings, J. Phys. B: At. Mol. Opt. Phys. 46, 175401 (2013) (http://klnrt.de/406); and in the mathematics by H. Kleinert and V. Zatloukal, Green Function of the DoubleFractional Fokker-Planck Equation-Path integral and Stochastic Diffrential Equations, (http://klnrt.de/403). The lecture in Taipeh can also be seen followed directly under the address http://klnrt.de/talks/CastTaiw.pptx.

Acknowledgements I am grateful to P. Jizba, N. Laskin, M. Lewenstein, A. Pelster, and M. Zwierlein for useful comments, and to Fabio Scardigli, Luca Di Fiore, and Matteo Nespoli for their hospitality in Taipeh during their Workshop Horizons of Quantum Physics (http://www.quantumhorizons.org). 


\section{References}

1. See http://en.wikipedia.org/wiki/Black_swan_theory

2. See http://www.nytimes.com/2007/04/22/books/chapters/0422-1st-tale. html? pagewanted=all.

3. Contrasting the 1994 book by R.J. Herrnstein and C. Murray entitled "The Bell Curve" http://de.wikipedia.org/wiki/The_Bell_Curve.

4. See http://en.wikipedia.org/wiki/2010_Flash_Crash.

5. V. Fock, Physik. Zeits. Sowjetunion 12404 (1937).

6. R.P. Feynman, Phys. Rev. 80, 440 (1950); R. P. Feynman and A.R. Hibbs, Quantum Mechanics and Path Integrals (McGraw-Hill, New York, 1965).

7. H. Kleinert, Path Integrals in Quantum Mechanics, Statistics, Polymer Physics, and Financial Markets, World Scientific, Singapore, 2006.

8. H. Kleinert, Gauge Fields in Condensed Matter, Vol. I, World Scientific Publishing Co., Singapore, 1989.

9. F. Black and M. Scholes, J. Pol. Economy 81, 637 (1973).

10. W. Feller An Introduction to Probability Theory and Its Applications vol. 2, Wiley, New York, 1991; J.-P. Bouchaud and M. Potters, Theory of Financial Risks, From Statistical Physics to Risk Management, Cambridge U. Press, 2000. See also Ch. 20 in [7.

11. A travelling pedestrian salesman is a Gaussian random walker, as a jetsetter he becomes a Lévy random walker.

12. J. Einasto, (arXiv:1109.5580).

13. R. E. Angulo, V. Springel, S. D. M. White, A. Jenkins, C. M. Baugh, C. S. Frenk, (arXiv:1203.3216).

14. Du Jiulin, 2004 Europhys. Lett. 67, 893 (2004), Phys. Lett. A 329, 262(2004).

15. C. Nardini, S. Gupta, S. Ruffo, T. Dauxois, and F. Bouchet, J. Stat. Mech. (2012) L01002 (http://arxiv.org/pdf/1111.6833.pdf)

16. F. Boettcher, C. Renner, H.P. Waldl, J. Peinke, (physics/0112063).

17. P. Bhattacharyya, A. Chatterjee, B.K. Chakrabarti, Physica A 381, 377 (2007) (physics/0510038)

18. S. Umarov, C. Tsallis, M. Gell-Mann, and S. Steinberg (http://arxiv.org/abs/cond-mat/0606040).

19. H. Kleinert, Phys. Rev. D 57, 2264 (1998) (cond-mat/9801167).

20. J.A. Lipa, J.A. Nissen, D. A. Stricker, D.R. Swanson, and T.C.P. Chui, Phys. Rev. B 68, 174518 (2003); M. Barmatz, I. Hahn J.A. Lipa, R.V. Duncan, Rev. Mod. Phys. 79, 1 (2007). Also see picture on the titlepage of the textbook 24].

21. H. Kleinert, Phys. Lett. A 277, 205 (2000) (cond-mat/9906107); Phys. Rev. D 60, 085001 (1999) (hep-th/9812197).

22. H. Kleinert, Multivalued Fields in Condensed Matter, Electromagnetism, and Gravitation, World Scientific, Singapore, 2008, pp. 1-497 (http://klnrt.de/b11).

23. V.N. Gribov and A.A. Migdal. Soviet Phys. JETP 28, 7841 (1969); See also H.D. Abarbanel, J.D. Bronzan, R.L. Sugar, and A.R. White, Phys. Reports 21, 119 (1975) [in particular Eq. (4.27)].

24. H. Kleinert and V. Schulte-Frohlinde, Critical Properties of $\phi^{4}$-Theories, World Scientific, Singapore 2001 (klnrt.de/b8).

25. In two dimensions, a sequence of critical exponents have been tabulated in A.A. Belavin, A.M. Polyakov, and A.B. Zamolodchikov, Nucl. Phys. B 241, 333 (1984).

26. M. Gell-Mann and F. E. Low, Phys. Rev. 95, 1300 (1954).

27. For the so-called Riesz fractional derivative see R. Metzler, E. Barkai, J. Klafter, Phys. Rev. Lett. 82, 3564 (1999); B.J. West, P. Grigolini, R. Metzler, and T.F. Nonnenmacher, Phys.Rev. E 55, 99 (1997). For the so-called Weyl derivative. See R.K. Raina and C.L. Koul, Proc. Am. Math. Soc. 73, 188 (1979): $\hat{p}_{4}^{1-\gamma} f(t) \equiv \Gamma^{-1}[1-\gamma] \int_{t}^{\infty} d t^{\prime}\left(t-t^{\prime}+i \epsilon\right)^{-2+\gamma} f\left(t^{\prime}\right)$.

28. The relevant functional matrix is $\left\langle\mathbf{x}\left|\left(-\nabla^{2}\right)^{\lambda / 2}\right| \mathbf{x}^{\prime}\right\rangle=$ $\Gamma[-\lambda / 2]^{-1} \int d \sigma \sigma^{-\lambda / 2-1}(4 \pi \sigma)^{-D / 2} e^{R^{2} / 4 \sigma}={ }^{D} c_{\lambda} R^{-\lambda-D}, \quad$ where $\quad{ }^{D} c_{\lambda}=$ 
$2^{\lambda} \Gamma((D+\lambda) / 2) / \pi^{D / 2} \Gamma(-\lambda / 2)$, and $R \equiv\left|\mathbf{x}-\mathbf{x}^{\prime}\right|$. If $\lambda$ is close to an even integer, it needs a small positive shift $\lambda \rightarrow \lambda_{+} \equiv \lambda+\epsilon$ and we can replace $\epsilon R^{\epsilon-1} / 2$ by $\delta(R)=S_{D} R^{D-1} \delta^{(D)}(\mathbf{R})$. For $A>0$ we have $\left|\mathbf{x}^{\prime}\right|^{-A}={ }^{D} c_{\lambda_{A}}^{-1}\left\langle\mathbf{x}^{\prime}\left|\left(-\nabla^{2}\right)^{\lambda_{A} / 2}\right| \mathbf{0}\right\rangle$ with $\lambda_{A} \equiv A-D$, so that we find $\int d^{D} x^{\prime}\left\langle\mathbf{x}\left|\left(-\nabla^{2}\right)^{\lambda / 2}\right| \mathbf{x}^{\prime}\right\rangle\left|\mathbf{x}^{\prime}\right|^{-A}=$ ${ }^{D} c_{\lambda_{A}}^{-1}\left\langle\mathbf{x}\left|\left(-\nabla^{2}\right)^{(\lambda+A-D) / 2}\right| \mathbf{0}\right\rangle={ }^{D} c_{\lambda+A-D}{ }^{D} c_{\lambda_{A}}^{-1}|\mathbf{x}|^{-A-\lambda}$.

29. Jun-Sheng Duan, J. Math. Phys. 46, 013504 (2005).

30. C. Fox, Trans. Amer. Math. Soc. 98, 395 (1961).

31. This technique is explained in Chapters 12 and 19 of Ref. 7]. The pseudotime $s$ resembles the so-called Schwinger proper time used in relativistic physics.

32. N. Laskin, (arXiv/1009.5533); Phys.Lett. A 268, 298 (2000); Phys. Rev. E 62, 3135 (2000); (ibid.) E 66, 056108 (2002); Chaos 10, 780 (2000); Communications in Nonlinear Science and Numerical Simulation 12, 2 (2007).

33. There should be no danger of confusing the fluctuating noise variable $\eta$ in this equation with the constant critical exponent $\eta$ in (9).

34. See Eq. (29.165) in Ref. 7.

35. I.H. Duru and H. Kleinert, Phys. Lett. B 84, 30 (1979) (klnrt.de/65/65.pdf); Fortschr. Phys. 30, 401 (1982) (klnrt.de/83/83.pdf). See also Chaps. 13 and 14 in [7].

36. A. Young and C. DeWitt-Morette, Ann. Phys. (N.Y.) 169, 140 (1984); H. Kleinert and A. Pelster, Phs. Rev. Lett. 78, 565 (1997);

37. L.Z.J. Liang, D. Lemmens, and J. Tempere, (arxiv:1101.3713)

38. For a pedagogical discussion see M. Zwierlein, MIT PhD Thesis 2006 (klnrt.de/papers/ZW.pdf)

39. We ignore the problem that near the resonance it is hard to confine the particles to the trap. See J. Stenger, S. Inouye, M.R. Andrews, H.-J. Miesner, D.M. Stamper-Kurn, and W. Ketterle, Phys. Rev. Lett. 82, 2422 (1999)

40. For the universal use of this functional see H. Kleinert, Fortschr. Phys. 30, 187 (1982) (klnrt.de/82).

41. See Eq. (1.35) in Ref. [24].

42. V. Bretin, V.S. Stock, Y. Seurin, F. Chevy, and J. Dalibard, Phys. Rev. Lett. 92, 050403 (2004).

43. L.P. Pitaevskii and S. Stringari, Bose-Einstein Con densation, Clarendon Press, Oxford, 2003; V.I. Yukalov, Laser Phys. 19, 1 (2009) (arXiv:0901.0636).

44. H.J. Schulz, Fermi liquids and non-Fermi liquids, in Proceedings of Les Houches Summer School LXI, ed. by E. Akkermans, G. Montambaux, J. Pichard, et J. Zinn-Justin (Elsevier, Amsterdam, 1995), p.533 (arXiv:condmat/9503150).

45. The decimal numbers are from seven-loop calculation in $D=3$ dimensions in Table 20.2 of Ref. 24].

46. See Eq. (10.191) in Ref. 24] and expand $f\left(t / M^{1 / \beta}\right) \sim \tilde{f}\left(\xi \Phi^{2 /(D-2+\eta)}\right)$ like $\tilde{f}(x)=1+c x^{-\omega}+\ldots$.

47. S.E. Pollack, D. Dries, R.G. Hulet, K.M.F. Magalhaes, E.A.L. Henn, E.R.F. Ramos, M.A. Caracanhas, V.S. Bagnato, Phys. Rev. A 82, 020701(R) (2010)

48. T. Donner, S. Ritter, T. Bourdel, A. Öttl, M. Köhl, and T. Esslinger, Science 315, 1556 (2007).

49. F. Dalfovo, S. Giorgini, P. Pitaevskii, and S. Stringari, Rev. Mod. Phys. 71, 463512 (1999) (Fig. 3); L.V. Hau, B.D. Busch, C. Liu, Z. Dutton, M.M. Burns and J.A. Golovchenko, Phys. Rev. A 58, R54 (1998) (Fig. 2).

50. H.D. Abarbanel and J.D. Bronzan, Phys. Rev. D 9, 3304 (1974). See their Eq. (40) 